\title{
Innovative Opportunities for Civility: Professional Development in a Time of COVID-19
}

Angela G. Opsahl, Jennifer L. Embree, and Matthew S. Howard

\begin{abstract}
The impact of the COVID-19 pandemic on nursing professional development has spurred innovative teaching efforts by educators. The application of new technology provided innovative support for participant engagement and allowed for evaluation of civility education learning outcomes. A technology expert provided necessary support in a live virtual environment. A planned dress rehearsal prior to the live event ensured the functionality of the virtual platform. The versatility of the new technology allowed breakout rooms and interactive software applications. Nursing professional development practitioners can use these tips to reimagine other face-to-face educational activities into a virtual platform.
\end{abstract}

\section{Introduction}

The influence of the COVID-19 pandemic has forced extreme disruption to daily lives and caused health professionals to transform how education supports quality and safety initiatives to the frontline staff and nursing students who care for patients. Educators, both in practice and academia, face extraordinary challenges to develop the next generation of health care providers in a pandemic (Rose, 2020). With social distancing requirements, the educational landscape has rapidly transitioned from face-to-face education to a virtual platform to drive ongoing educational efforts.

\section{Health Care Education}

Priorities in health care education rapidly transitioned to support learners and organizations during the pandemic. Learner welfare became an essential component in the educational activity planning process. Nurse educators were suddenly required to deploy suitable technologies to ensure ease of access, provide discussion points for reflection, and maintain flexibility for learners (Anderson et al., 2020).

Prepandemic planning began on a face-to-face educational activity featuring an academic-practice partnership collaboration. The stress of COVID-19 exacerbated the possibilities of uncivil responses and behaviors, making this workshop more important. The planning committee consisted of nurse administrators, clinical nurse educators, and nursing faculty. The educational program centered on civility in the health care setting to improve quality and safety. Incivility can have a destructive effect on patient outcomes, safe practices, moral courage, and staff turnover rates (Clark, 2019). This academicpractice partnership educational activity on civility was the first of future educational offerings intending to influence current and future local health care education.

\section{Professional Development Planning}

Due to safety concerns, the planning committee was charged with transforming the face-to-face educational activity into a virtual event. Additional and more frequent planning committee meetings

This is the author's manuscript of the article published in final edited form as: 
were necessary to review potential issues involved in a rapid transition from face-to-face to a virtual event (Embree \& Little, 2020). The potential technology platforms from both the university and the local hospital were assessed. The planning committee identified the need to use technology that provided ease of access and availability for the learner with small-group discussions (Table 1). Capitalizing on the academic partnership, use of the university Zoom webinar technology and the Zoom meeting technology was investigated. Other platforms were available, but as this was a low-budget educational activity, selecting a current technology platform made fiscal sense.

With the complexity of various technology platforms, a technology expert from the university was consulted and included on the planning committee. This additional support was necessary when using two types of virtual software during the educational activity and webinar meeting. The technology expert assisted in the rapid transition of the event to a virtual platform.

All presenters were asked to have their content material completed and submitted to a secured group cloud-based storage file 1 week before the educational activity. The planning committee then created a finalized agenda and reviewed the presentation format for continuity. Prior to the educational activity, a rehearsal was planned for the presenters and technology expert to work through all content to decrease presenter anxiety and improve technology familiarity. This rehearsal provided an opportunity for all presenters to practice their presentations' content to ensure the platform functioned appropriately and to be more comfortable with the educational event platforms. In addition to the primary virtual platform, breakout rooms were implemented to allow for small-group discussions. Each small group would then provide a summary of their discussion to the full conference of participants.

In addition to the webinar and meeting platform, the planning committee decided to use an additional software platform to engage conference participants in real-time. Although other platforms were available, Menti-meter was selected by the committee to add other mechanisms of engagement. This interactive presentation overlay tool allowed users to answer polling questions and open-ended questions to develop word clouds. These features enriched the content and discussion. Pre- and posttest surveys were created, and links to the surveys were added to the finalized presentation to ease participant access. The multiple technologies supported optimal learning and assessment with several opportunities for participant engagement during the activity.

\section{Results}

The planning committee deemed the educational event a success after reviewing university faculty, staff nurses, nursing students, and nurse educators' feedback. The responses were overwhelmingly positive. One conference participant stated, "The event was a great collaboration between nurse administrators, nurse educators, and faculty." Another participant noted, "The entire educational workshop was so interactive and fun to experience." Many participants found the inclusion of the interactive software beneficial to their learning process and engagement prompting one participant to state, "[The] interactive technology allowed us to engage the nurses and nursing students fully in the content."

\section{Conclusion}


Given the challenges educators face during the COVID-19 pandemic, adaptability and flexibility are vital in forging ahead in educational initiatives to prepare current and future clinicians. Virtual platforms with innovative technology allow educators to offer educational content to engage participants while maintaining pandemic social distancing requirements. The alteration to our educational environment offered unique opportunities to change how we offered a professional development activity to support the local community for years to come.

\section{References}

Anderson, M. L., Turbow, S., Willgerodt, M. A., \& Ruhnke, G. W. (2020). Education in a crisis: The opportunity of our lives. Journal of Hospital Medicine, 15(5), 287-289.

https://doi.org/10.12788/jhm.3431

PMID:32379031

Clark, C. M. (2019). Fostering a culture of civility and respect in nursing. Journal of Nursing Regulation, 10(1), 44-52. https://doi.org/10.1016/S2155-8256(19)30082-1

Embree, J. L., \& Little, A. (2020). Using technology to provide socially distanced professional development and continuing education. The Journal of Continuing Education in Nursing, 51(8), 355358. https://doi.org/10.3928/00220124-20200716-04

PMID:32722812

Rose, S. (2020). Medical student education in the time of COVID-19. Journal of the American Medical Association, 323(21), 2131-2132. https://doi.org/10.1001/jama.2020.5227

PMID:32232420 
Table 1

Teaching Tips for Moving a Face-To-Face Workshop into a Virtual Event

1. Assemble the team, considering what stakeholders need to be included due to the delivery method change.

2. Investigate what video communication or conferencing platform for technology is available and affordable to use.

a. Identify software or platforms that provide participant-friendly interactivity.

3. Include technology experts on the planning committee of the virtual educational activity.

4. Test the technology through a rehearsal with all presenters.

5. Identify a participant-friendly software or platform to administer the educational activity evaluation. 